\title{
Spatial resolutions and field-of-views in millimetre wave aperture synthesis security screening imagers
}

\author{
Neil A. Salmon \\ Manchester Metropolitan University, Manchester, M15 6BH, UK
}

\begin{abstract}
Aperture synthesis for passive millimetre wave imaging provides a means to screen people for concealed threats in the extreme near-field configuration of a walk-through portal; a regime where the imager to subject distance is of the order of both the required depth-of-field and the field-of-view. As shown here, the field-of-view in these scenarios is unlimited and the spatial resolution is slightly better than the half-wavelength Abbe microscope resolution. As in this scenario the subject would be surrounded by receiving antennas, all surfaces of the human body will be in focus with high-resolution, even at the low frequencies. For example at a frequency of $22.51 \mathrm{GHz}$ the spatial resolution is in the region of $\sim 7 \mathrm{~mm}$. This capability is ideally suited to the aircraft security screening industry. Furthermore, the aperture synthesis imager is well suited to non-cooperate screening at longer ranges, as the antenna arrays may be deployed on almost any available surface. The field-of-view of these systems is likewise unlimited, extending from the near-field to the far-field. The spatial resolution of these systems, as here shown, is in the region of the half-wavelength resolution close to the imager, and slightly better than the Fraunhofer diffraction resolution limit at longer ranges. Therefore, also for the non-cooperate screening scenarios, the subject will always be in focus with the best possible spatial resolution allowed by the physics of imaging.
\end{abstract}

Keywords: Aperture synthesis, imaging, security screening portal, spatial resolution, field-of-view, three-dimensional, millimetre wave, terahertz, near-field

\section{INTRODUCTION}

There is a continued drive to have ever improved security screening systems in society. The millimetre wave band has attractive attributes to this end, as clothing is almost transparent to this radiation [1],[2]. This means that any millimetre wave imaging system will be able to see the surface of the human body, as millimetre wave only penetrate a fraction of a millimetre into the skin. Furthermore, as the signatures of human skin are well known, screening systems have the potential to identify anomalies present under the clothing [3],[4].

Although the capabilities of millimetre wave imaging where revealed during the 1980's and early 1990's by imaging systems that used lenses and mirror [5], their capabilities were limited for portal screening of people [6]. This is because in such scenarios the imager to subject distance is of the order of half a metre and the field-of-view needs to be that of the subject height, typically $<2 \mathrm{~m}$. Lens or mirror based millimetre wave imagers have a depth-of-field fundamentally limited to around $10 \mathrm{~cm}$ to $20 \mathrm{~cm}$ by the optical aberrations in the focussing lenses and mirrors, even in well optimised systems. Because of this, active (radar) millimetre wave imagers started to dominate the portal screening market since year 2000, as they were not limited by depth-of-field problems [7]. However, radar systems suffer illumination problems associated when imaging human body canyon regions (eg. between the legs) and image reconstruction problems where there is multi-path reflection from different body surfaces. Fortunately, an aperture synthesis passive millimetre wave imaging system has all of the benefits of these two system and none of the disadvantages, so is ideally suited to portal and non-cooperate security screening. Furthermore, as passive (or radiometric) imaging systems do not suffers speckle effects (as it images incoherent radiation) the systems can deliver the full theoretical spatial resolutions possible.

\section{GENERIC FOURIER TRANSFORM IMAGE GENERATION ALGORITHM}

* Correspondence: Email: n.salmon at mmu.ac.uk; Telephone: +44 7921172892 
The aperture synthesis imaging capability exploits the coherence properties of radiometrically generated radiation. Just how this can lead to an imaging capability is far from obvious, but the technique has been rigorously demonstrated by the radio astronomers for a number of decades by creating two-dimensional images of astrophysical sources. When applying this technique to the near-field it enables a three-dimensional (3D) imaging capability, providing the Abbe microscope, half-wavelength spatial resolution, as outlined in [1]. An ideal application of this is for a security screening portal for the aviation security industry.

The technique exploits the fact that the cross-correlation $\left(V_{\mathrm{J}, \mathrm{K}}^{,}\right)$given by

$$
V_{J, K}^{\prime}=\left\langle v_{J}(t) v_{K}^{*}(t)\right\rangle
$$

of the electric fields $\left(v_{\mathrm{J}}\right.$ and $v_{\mathrm{K}}$ ) at two locations in space, having position vectors $\mathbf{R}_{\mathrm{J}}$ and $\mathbf{R}_{\mathrm{K}}$, from the radiometric emission from an object, represents one Fourier component of the spatial frequencies of the image of that object. This means that if sufficient numbers of spatial frequencies can be collected, an inverse Fourier transform of these can be used to create the image. This Fourier transform relationship between the cross-correlations and the image is one in three-dimensions and it is referred to as the van Cittert Zernike theorem [8]. The discretised inverse transform of this, used to generate the three-dimensional image is given by

$$
I(\mathbf{l})=[A(\mathbf{l})]^{-1} \sum_{J, K, J \neq K}^{n(n-1) / 2} V\left(\mathbf{u}_{J, K}\right) \exp \left(i \mathbf{u}_{J, K} \mathbf{l}\right)
$$

where $\mathbf{l}$ is the direction cosine vector of the image voxel from the phase centre, $\mathbf{u}_{\mathrm{J}, \mathrm{K}}$ is the spatial frequency vector and $V_{J, K}\left(\mathbf{u}_{\mathrm{J}, \mathrm{K}}\right)$ is referred to as the visibility function and contains the phase-corrected cross-correlations defined by

$$
V_{J, K}\left(\mathbf{u}_{J, K}\right)=V_{J, K}^{\prime} \exp \left(-i \phi_{J, K, C A L}\right) \text {. }
$$

This phase correction is effectively a phase calibration of the cross-correlations and results in centring the $3 \mathrm{D}$ image at a defined phase centre in space, having a position vector $\mathbf{R}_{\mathrm{PC}}$. The phase calibration factor $\phi_{\mathrm{J}, \mathrm{K}, \mathrm{CAL}}$ can be determined by measuring a noise source placed at the phase centre, or at an adjacent defined location. The spatial frequency in three dimensions, by definition [9], is represented by the vector

$$
\mathbf{u}_{J, K}=\nabla \phi_{J, K}
$$

where $\phi_{\mathrm{J}, \mathrm{K}}$ is the phase of the cross-correlation (a scaler function) from a source at the phase centre of the image and $\nabla$ is nabla, the spatial gradient operator. Vector calculus on the last equation indicates the spatial frequency vector abscissa in $3 \mathrm{D}$ space is given by

$$
\mathbf{u}_{J, K}=\frac{2 \pi}{\lambda}\left(\frac{\mathbf{R}_{J}-\mathbf{R}_{P C}}{\left|\mathbf{R}_{J}-\mathbf{R}_{P C}\right|}-\frac{\mathbf{R}_{K}-\mathbf{R}_{P C}}{\left|\mathbf{R}_{K}-\mathbf{R}_{P C}\right|}\right)
$$

There are two possible routes to determining the image from the Fourier transform of Eq. 2. The first of these is the analytic Fourier transform, referred to by the radio astronomers as the direct analytic Fourier transform [11], which is the transformation of in Eq.2. The benefit of this is that there is no intermediate processing of data that may lead to image artefacts, but the disadvantage is that computationally it can be quite intensive, particularly if there are many receivers and voxels in the image. This is compounded by the fact that for $n$ receivers, there will be $n(n-1) / 2$ complex crosscorrelations. Given the complexity of Fourier transforms by this method rises as the square of the number of points, the complexity of the transform is proportional to $n^{4}$.

The second route is referred to as the Fourier transform gridding route. Here a regular grid in 3D visibility space is set out on to which the phase corrected cross-correlations, $V_{J, K}$ are interpolated. With the cross-correlations interpolated to the regular grid a discreet Fourier transform (DFT) can be used, which is then calculated using the Fast Fourier Transform (FFT). This has the advantage of being extremely quick for the computation, but it does introduce small errors arising from the interpolation on to the regular grid. Using the FFT the complexity of the transform reduces, from $n^{4}$ for the analytic transform, to $\sim 2 n^{2} \log (n)$. So the FFT route is a factor of $n^{2} /(2 \log (n))$ faster than the DFT route.

\section{FIELD-OF-VIEW AND SPATIAL RESOLUTION BY SIMULATION}


The limits on field of view and the spatial resolution in aperture synthesis images are estimated by simulation. Delta functions acting as sources are used to generate a set of cross-correlations as they would be measured by an antenna array and a cross-correlator, using the method outlined in [1]. These are then placed on a spatial frequency abscissa as estimated by Eq. 5 and then Fourier transformed to generate the image as indicated in the previous section.

In the case of the field-of-view, three equi-spaced delta functions in a line are place at the centre of the scene. The crosscorrelations that these generate are then Fourier transformed to create the image. The imaged is examined to determine if the three delta functions are faithfully reproduced in the image by noting their amplitudes. The distance between the delta functions is gradually increased until the amplitude of the outer two delta functions becomes half the amplitude of the central delta function. When this situation has been achieved the spacing between the two outer delta function in the scene defines the field-of-view. This scenario is illustrated in Figure 1.

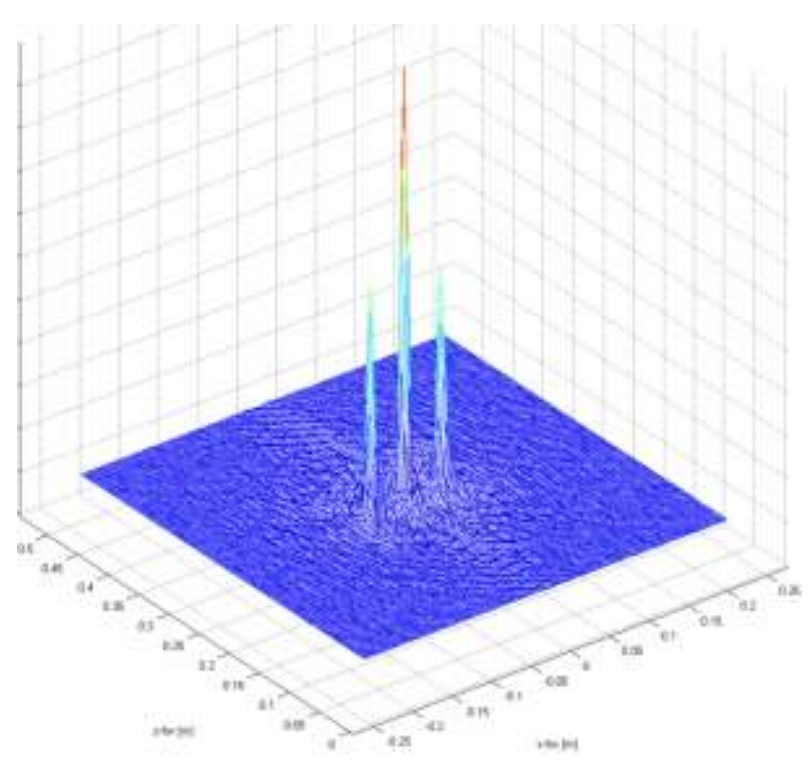

Figure 1. The three-delta function in a line used to estimate the field-of-view of the imager

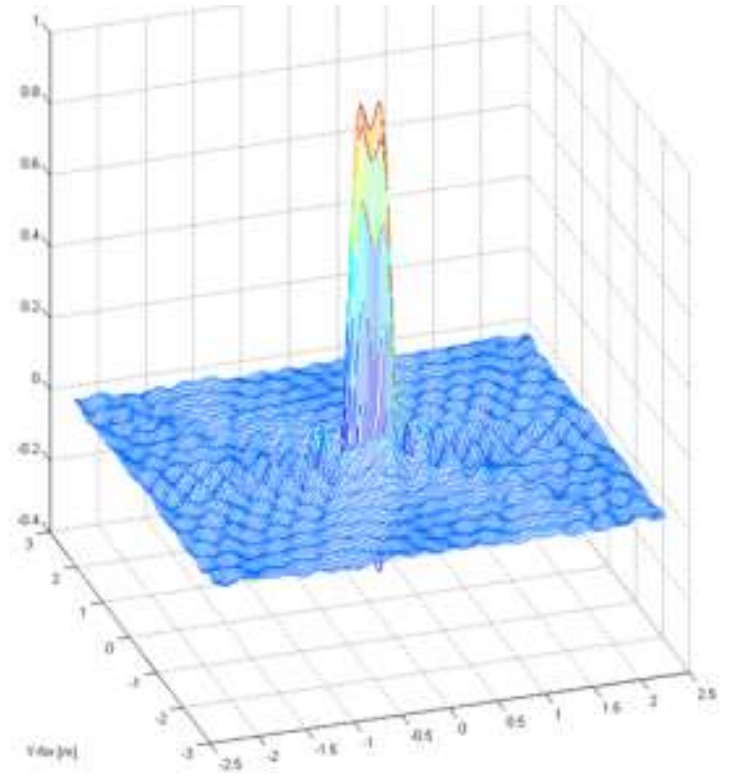

Figure 2. The two delta functions are used to estimate the spatial resolution. Seen here they are only just resolvable.

In the case of the spatial resolution a similar analysis is performed using two delta functions. Two delta functions are place close together in the centre of the scene. When the two delta functions are far apart they can be seen as two distinct sources in the image. When they are closer than the resolution limit they are no longer discernible as two separate sources. The distance apart at which the two sources are barely discernible as two separate sources defines the Sparrow limit of the resolution [10]. This is a good spatial resolution metric, as it closely assimilates the sensitivity to intensity of the human eye. At the Sparrow limit the gradient of the intensity across the top region of the image is zero, and the spatial change in the gradient is also zero, ie the intensity variation between the locations of the delta functions in the image is flat. The two delta functions shown in Figure 2 are only just resolvable as two separate sources.

Two different arrays of antennas have been simulated, both having in the region of 340 receiving antennas with a $0 \mathrm{dBi}$ gain. The first type of antenna array is flat and circular having diameters of $0.3 \mathrm{~m}$ and $0.6 \mathrm{~m}$, as illustrated in Figure 3 . This diameter has been chosen as it may be close to that of a next demonstrator system. The final walk-through portal screening would use antennas on either side and above and below the subject, in order that all surfaces of the human body would be measured. However, to progress to this stage an intermediate demonstrator imager would be required generating $\sim 10 \mathrm{k}$ pixels, to de-risk the route and convince stake holders of the likely performance of a final system.

The second type of antenna is a circular array of inward looking antennas having a diameter also of $0.3 \mathrm{~m}$ and $0.6 \mathrm{~m}$. This architecture of imager was chosen as it enables a comparison with the flat antenna array system. In configuration this array also has some similarities to a walk-through archway system, although being considerably smaller. The walk- 
through archway system is envisaged to be $2 \mathrm{~m}$ high, with a $1 \mathrm{~m}$ wide walk-through. The two types of array are illustrated in Figure 3.
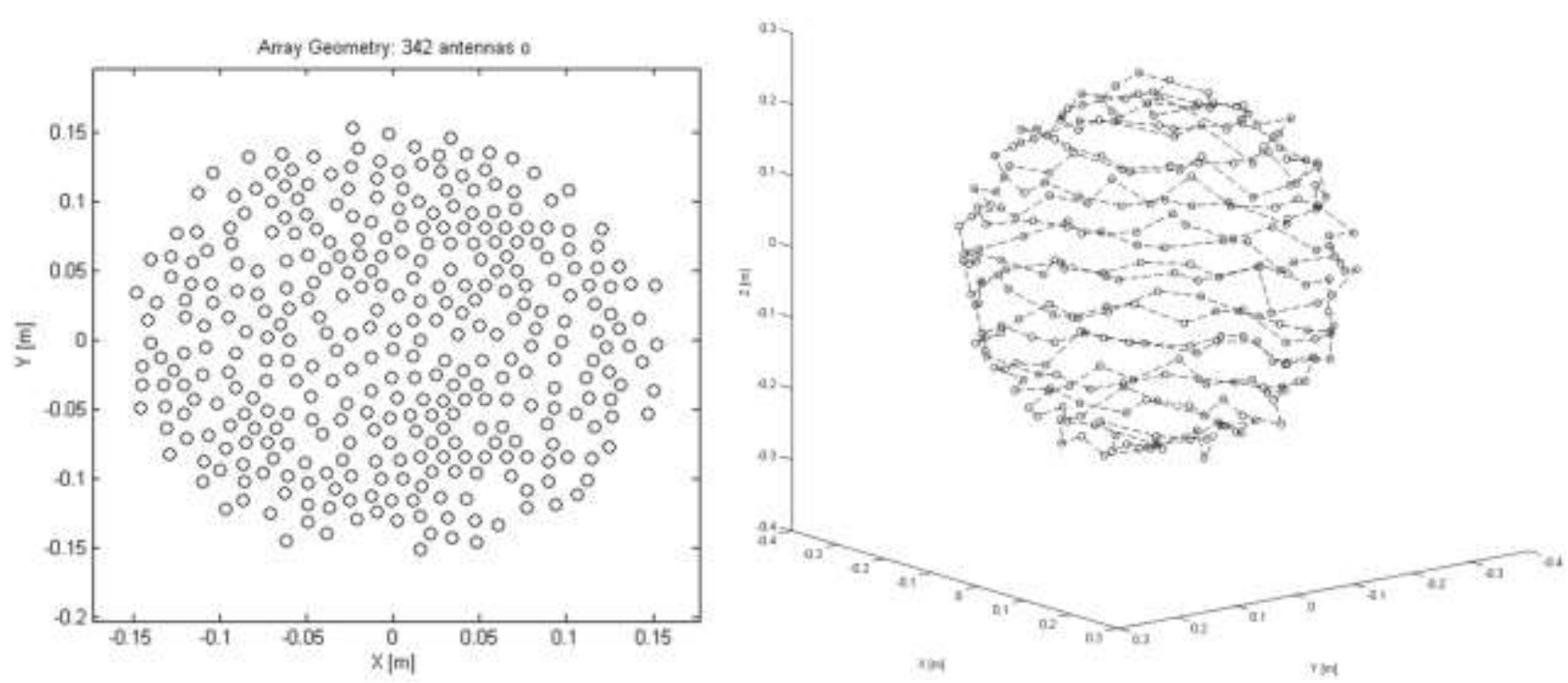

Figure 3. The two types of antenna array used in the simulation of the field-of-view and the spatial resolution, on the LHS a two-dimensional circular array of outward looking antennas and on the RHS spherical array of inward looking antennas. Antenna positional locations are randomised in space to minimise the effects of aliasing.

Radiation frequencies that were chosen for simulation were $3.71 \mathrm{GHz}, 22.51 \mathrm{GHz}$ and $320 \mathrm{GHz}$. These frequencies were chosen as aperture synthesis systems at these frequencies have a number of benefits. For example, at $3.71 \mathrm{GHz}$ the spatial resolution would be in the region of $4 \mathrm{~cm}$, so at these low frequencies there would be a much smaller number of antennas, so this might be the basis of a low cost walk-through demonstrator portal. At a frequency of $22.51 \mathrm{GHz}$ this would provide about $7 \mathrm{~mm}$ of spatial resolution in a walk-through portal for airport security screening. It is also a frequency where there are existing receiver designs and the costs of components would be relatively low. The frequency of $320 \mathrm{GHz}$ is just about the highest frequency that would be used for security screening, without getting into problem associated with scattering from clothing. These higher frequency systems might be useful for longer range as they would deliver higher spatial resolutions, but their costs would be considerably higher.

\section{THE FIELD OF VIEW FROM A SINGLE FOURIER TRANSFORM IMAGE}

The difficulty with the Fourier transform route to generating images is that in moving from the far-field regime to the near field regime the relationship between the scene and the visibility function deviates from that of a Fourier transform. As far as creating an image from the visibility function, this is possible in the near-field regime, defined as being those ranges shorter than the Rayleigh distance $2 D^{2} / \lambda$, where $D$ is the aperture size in metres. However, in moving into the extreme near-field images become increasingly distorted and the useable field of view shrinks compared to that in the far-field. The effect of this shrinking of the field of view is therefore estimated in this paper by simulation.

The fields-of-views estimated by simulation as a function of range for two-dimensional circular arrays having diameters of $0.3 \mathrm{~m}$ and $0.6 \mathrm{~m}$ are shown in Figure 4 . These simulations indicate that the field-of-view increases weakly with range out to a range of $0.2 \mathrm{~m}$ and $0.3 \mathrm{~m}$ for the $0.3 \mathrm{~m}$ and $0.6 \mathrm{~m}$ diameter arrays respectively. Beyond these ranges the field of view rises almost linearly with range. The Rayleigh distances $2 D^{2} / \lambda$ for these two arrays are $13.5 \mathrm{~m}$ and $54.1 \mathrm{~m}$ respectively, so this indicates the field-of-view is not limited by the Fourier transform until ranges much closer than the Rayleigh distance are reached. For comparison, two Fresnel scales $(2 \sqrt{ }($ range. $\lambda)$ are plotted in the figure. In the region where the field-of-view starts to vary linearly with range the field-of-view almost equals that of two Fresnel Scales. At all other ranges the field-of-view are larger. Goodman [9] indicates the field-of-view in the near-field is in the region of four Fresnel scales. 

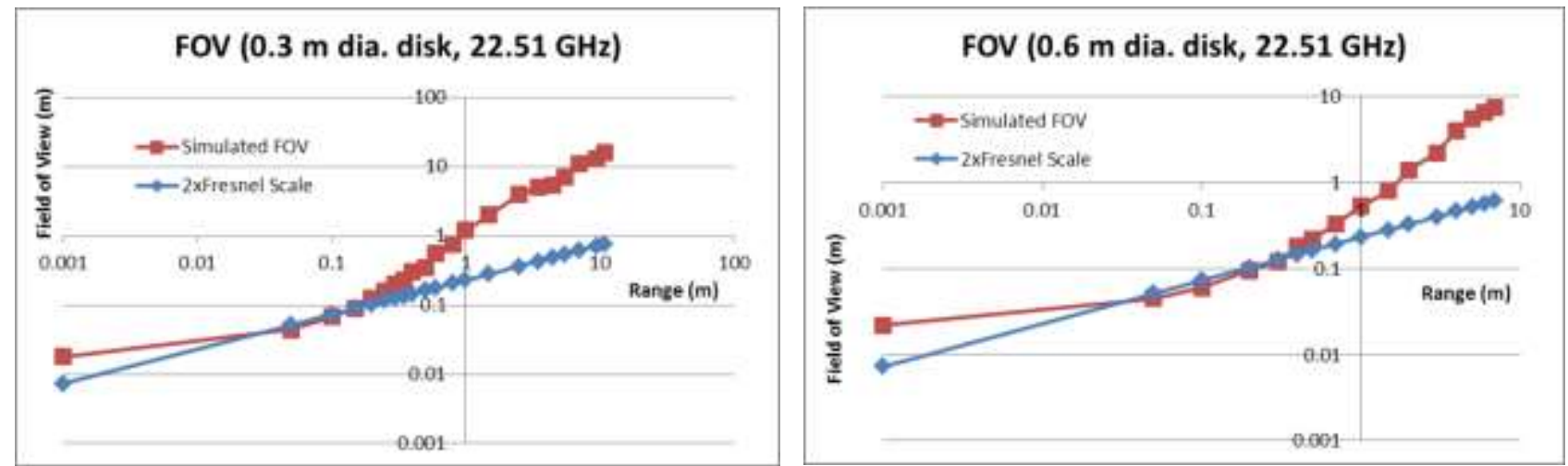

Figure 4: The variation in the field-of-view (FOV) estimated using the three-delta function technique and two Fresnel scales as a function of range at $22.51 \mathrm{GHz}$ for circular two-dimensional arrays having diameters of $0.3 \mathrm{~m}$ and $0.6 \mathrm{~m}$.

The fields-of-views estimated by simulation on the inside of the spherical array of antennas having diameters of $0.3 \mathrm{~m}$ and $0.6 \mathrm{~m}$ are shown in Figure 5. These simulations indicate that the field-of-view are more or less constant and uniform inside the sphere of antennas in both the $\mathrm{x}$ and $\mathrm{z}$-direction. However, the $\mathrm{z}$-field-of-view increases slightly when the $\mathrm{z}$ range approaches the spherical surface of the antennas. The field-of-view is close to the root of the product of the array diameter size and wavelength, as indicated in the figure.
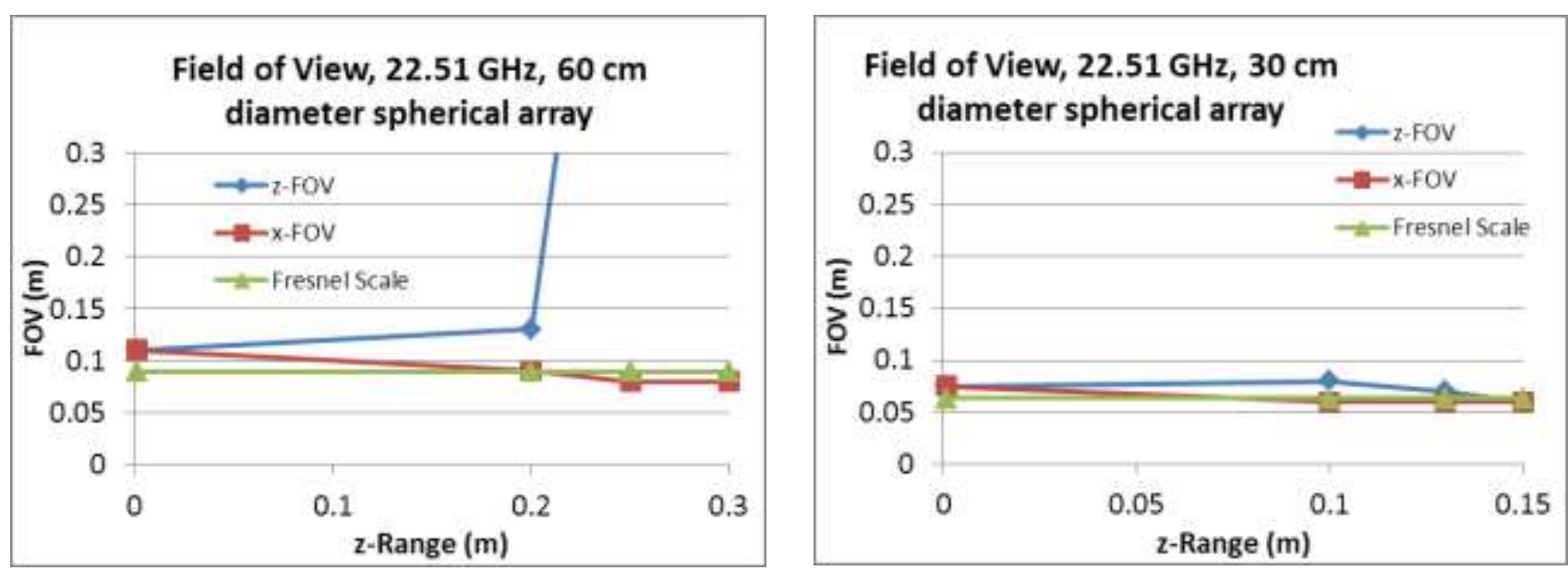

Figure 5: The variation in the field-of-view estimated using the three-delta function technique in the $\mathrm{x}$ and $\mathrm{z}$ direction, together with the Fresnel Scale root (lambda*baseline).

Comparisons for the same sized antenna arrays at frequencies of $3.71 \mathrm{GHz}$ and $320 \mathrm{GHz}$ indicate there is a similar behaviour to those at $22.51 \mathrm{GHz}$. That is, for the two-dimensional circular arrays the field-of-view out to ranges of about the radius of the array is that set by the Fresnel scale, so is proportional to the root of the range. However, at longer ranges the field-of-view (in linear dimensions) is much larger than the Fresnel scale and is directly proportional to the range. In the case of the three-dimensional spherical arrays the field-of-view inside the sphere is constant at a size approximately given by the root of the product of the array diameter and the wavelength. A more precise scaling of this field-of-view (FOV) for the arrays sizes and frequencies indicate the following. This scaling is:

- $\quad \mathrm{FOV}=1.3 \mathrm{D}^{0.49} \lambda^{0.52}$ (for the frequency band $3.71 \mathrm{GHz}$ to $22.51 \mathrm{GHz}$ ),

- $\mathrm{FOV}=5.1 \mathrm{D}^{0.85} \lambda^{0.35}$ (for the frequency band $22.51 \mathrm{GHz}$ to $320 \mathrm{GHz}$ ),

for a spherical array of antennas having a diameter $D$, sensitive at a radiation wavelength of $\lambda$. This limit on the field-ofview from a single Fourier transform is relevant for both the analytic direct Fourier transform and the gridding FFT route to creating images. It is not a fundamental limit as to cover the full field-of-view of a security screening portal several 
Fourier transforms would need to be performed for different regions of space. So the image would be a mosaic of Fourier transforms images with different phase centres, with spacings given by the above field-of-view limits. The limit on spatial extend of a single Fourier transform does not restrict imager field-of-view of the G-matrix route to image creation. However, this route does mean the use of large matrices for high resolution systems with many antennareceivers, as the matrix dimension is the number of antenna-receivers by the number of pixels (or voxels).

This indicates that for a security screening portal the field-of-view can extend to cover any region in front of the antennas, this indicates the field-of-view of the aperture synthesis system is unlimited, which offers a great benefit over the lens and mirror based focussing systems.

\section{SPATIAL RESOLUTIONS OF IN THE SECURITY SCREENING PORTAL}

The results of estimating the sparrow resolution by simulation for a two-dimensional circular antenna array having diameters $D$ of $0.3 \mathrm{~m}$ and $0.6 \mathrm{~m}$ at $22.51 \mathrm{GHz}$ at $22.51 \mathrm{GHz}$ are shown in Figure 6 . These simulations indicate that close to the array the cross-range sparrow spatial resolution is $90 \%$ of the Abbe microscope resolution and that further away the resolution is $68 \%$ of the $1.22 \lambda / \mathrm{D}$ Fraunhofer diffraction limit based on the Rayleigh resolution criterion. The Sparrow resolution is therefore slightly more optimistic than the Rayleigh resolution, which is normally the case with imagers [10].
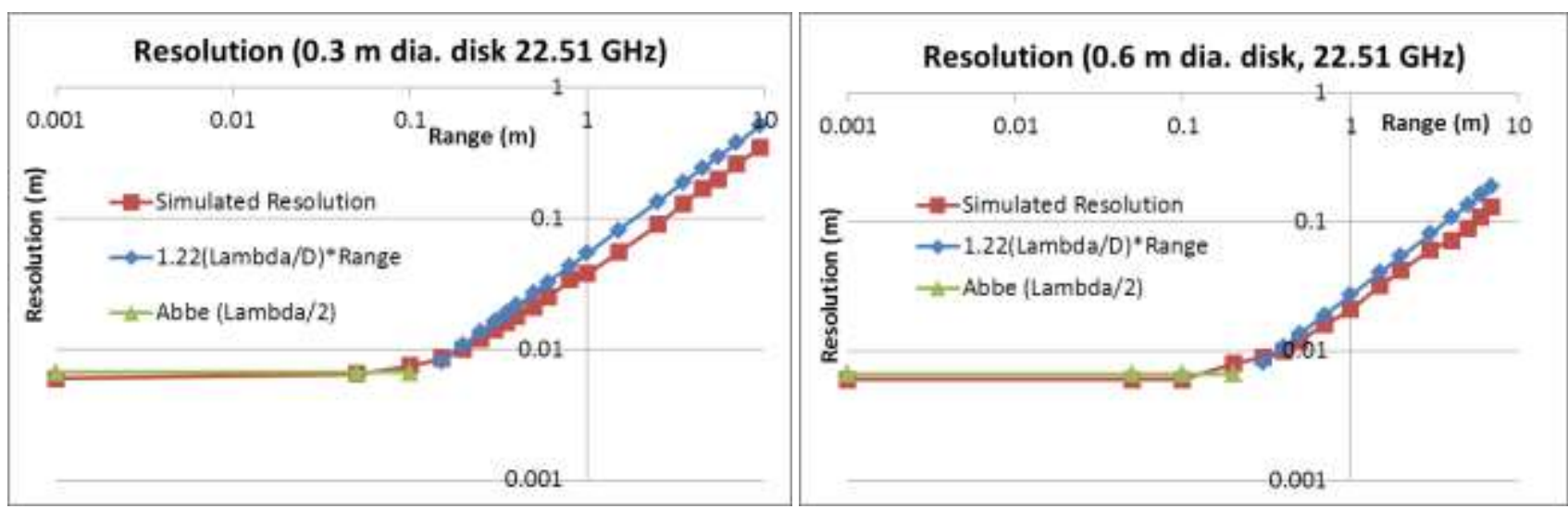

Figure 6: The variation in the (cross-range) sparrow spatial resolution as a function of range at $22.51 \mathrm{GHz}$ for circular two-dimensional antenna arrays having diameters of $0.3 \mathrm{~m}$ and $0.6 \mathrm{~m}$, estimated by simulation. At close range the resolution is slightly better than the Abbe microscope resolution and at longer ranges slightly better than the $1.22 \lambda / \mathrm{D}$ resolution from Fraunhofer diffraction.

The results of estimating the sparrow resolution by simulation for a three-dimensional spherical array having diameters of $0.3 \mathrm{~m}$ and $0.6 \mathrm{~m}$ at $22.51 \mathrm{GHz}$ are shown in Figure 7 . The sparrow resolution is $90 \%$ of the Abbe $(\lambda / 2)$ microscope resolution except in the immediate vicinity of the antennas, where it rises slightly.

Analysis of the $0.3 \mathrm{~m}$ and $0.6 \mathrm{~m}$ diameter circular and spherical arrays at the frequencies of $3.71 \mathrm{GHz}$ and $320 \mathrm{GHz}$ indicate a similar spatial resolution scaling to those of the above $22.51 \mathrm{GHz}$ systems. 

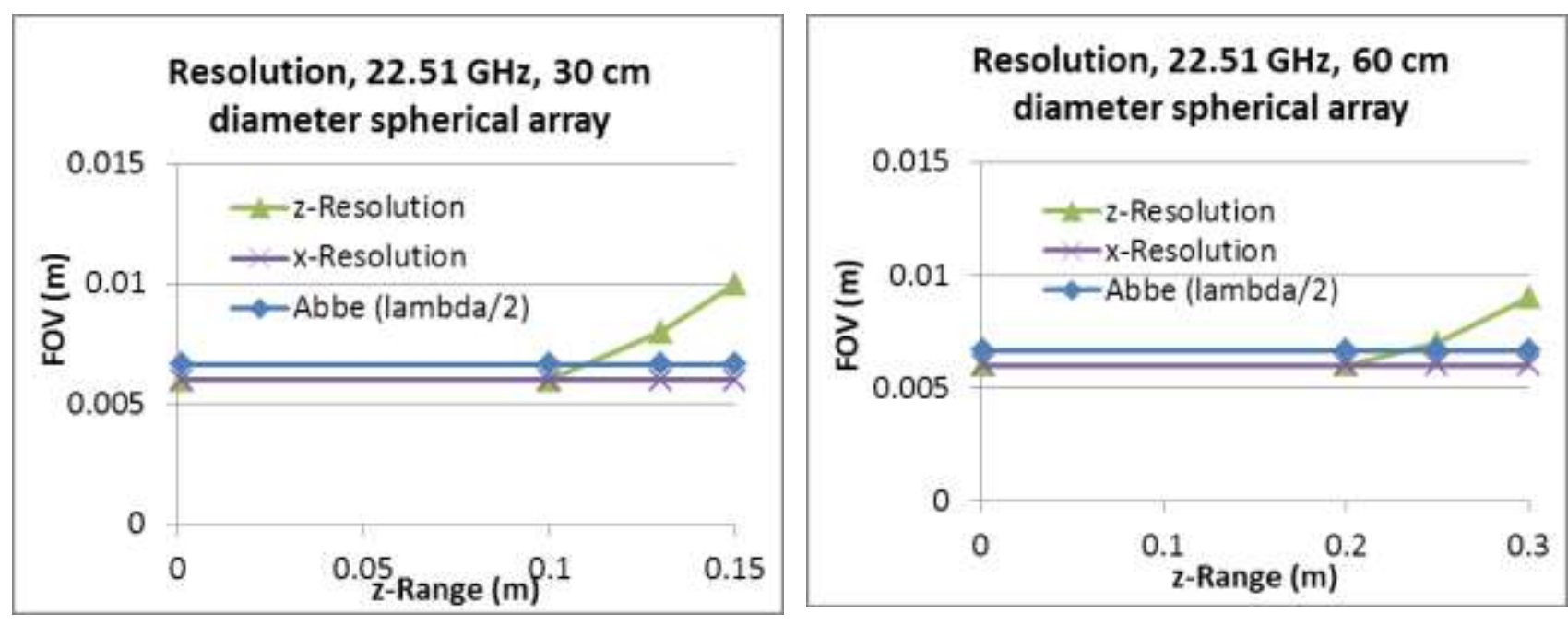

Figure 7: The variation in the (cross-range) sparrow spatial resolution at $22.51 \mathrm{GHz}$ as a function of distance from the centre of three-dimensional spherical antenna arrays having diameters of $0.3 \mathrm{~m}$ and $0.6 \mathrm{~m}$, estimated by simulation. Throughout the majority of the inside of the sphere of antennas the spatial resolution is slightly better than the Abbe microscope resolution.

This indicates if a security screening portal where developed where the subject were surrounded by antennas, the spatial resolution would always be close to half-wavelength, regardless of where in the volume the subject was located. This means that any region in front of the antennas can be imaged with a resolution that is just slightly better than the Abbe microscope resolution close to the antennas, or just slightly better than the Fraunhofer diffraction resolution at longer ranges.

\section{IMPLICATIONS FOR WALK-THROUGH AND NON-COOPERATE SCREENING}

There is great flexibility of passive aperture synthesis imaging systems for walk-through portal and non-cooperate screening as the field-of-view of these systems is unlimited and the spatial resolution is either the half-wavelength Abbe microscope resolution (in the near-field) or the Fraunhofer diffraction resolution $\lambda /$ (aperture size) (in the far-field), such a capability not possible with conventional lens or mirror based passive millimetre wave imagers. This happens because the phase information about the imagery is stored in the cross-correlations and can be accessed to focus an image anywhere by either Fourier transforms or the G-matrix technique. In the lens or mirror based systems the phase information is lost by the squaring process in the detectors of the focal plane array, so the image is in focus only at the location defined by the focussing elements, this fundamentally limiting the depth-of-field in the critical near-field regions of personnel security screening scenarios.

The flexibility offered by the aperture synthesis imaging system means that a walk-through security screening portal can be developed, as subjects will always be in focus as they walk through the portal. Furthermore, antennas arrays can be matched to deployment scenarios meaning they can "blend" into the infrastructure, enabling them to be unobtrusive, thus presenting a low-stress environment for law abiding citizens. In particular for non-cooporate screening, the aperture synthesis imager is well suited, as subjects at any distance from the array will be in focus, this being in a near-field or a far-field scenario.

The speed of image analysis for one-second transit time walk-through portals would be targeted by machine analysis of the 3D surface image of the human body, using a skin model of the human body to detect anomalies [3]. The reason for this is that there would be so much detail in the image, assimilation of this by a manual operator would just take too long. Machine analysis will greatly accelerate this screening process.

\section{FUTURE WORK}

The next phase of the technology development of aperture synthesis imaging would be the development of a $\sim 10 \mathrm{k}$ pixel near-field imaging system that would demonstrate imaging of non-metallic threats in human body canyon regions and areas that remain un-illuminated in active radar systems. In this system the Gerchberg algorithm would be demonstrated 
to deliver higher quality imagery [12]. This system would require the use of a low-cost cross-correlator capable of processing the outputs of hundreds of receiver channels with bandwidths of hundreds of megahertz as discussed in [13]. The follow-on to this would be a full body walk-through portal screening prototype for the detection of non-metallic threats anywhere on the human body.

\section{ACKNOWLEDGEMENTS}

The author gratefully acknowledges the partial financial support for this work from the Future Aviation Security Solutions (FASS) programme from the United Kingdom government.

\section{REFERENCES}

[1] Salmon, N. A.,"3-D Radiometric Aperture Synthesis Imaging”, IEEE TMTT, vol. 63, Issue 11, (2015).

[2] Salmon, N. A. Wilkinson, P.N. and Taylor, C., "Interferometric aperture synthesis for next generation passive millimetre wave imagers", SPIE Europe Security+Defence Europe, 'Millimetre Wave and Terahertz Sensors and Technology V', Edinburgh, September, (2012).

[3] Owda, A., Salmon, N.A., Rezgui, N-D, "Electromagnetic Signatures of Human Skin in the Millimeter Wave Band 80-100 GHz, Progress in Electromagnetic Research B 80, March, (2018).

[4] Owda, A. Salmon, N.A., Harmer, S., Shylo, S., Borwing, N.J., Rezgui, N-D, Shah, M., "Millimeter-Wave Emissivity as a Metric for the Non-Contact Diagnosis of Human Skin Conditions" Journal of Bioelectromagnetics, vol. 38, issue 7, pp.559-569, DOI: 10.1002/bem.22074, October, (2017).

[5] Appleby, R., Anderton, R.N., Salmon, N.A. "Compact real-time (video rate) passive millimeter-wave imager", Passive Millimeter-Wave Imaging Technology III, vol. 3703, SPIE (1999).

[6] Salmon, N.A., Bowring, N., Hutchinson, S., Southgate, M., O’Reilly, D., “An aviation security (AVSEC) screening demonstrator for the detection of non-metallic threats at 28-33 GHz", Millimetre Wave and Terahertz Sensors and Technology, VI, Dresden, Proc. SPIE vol. 8900, October, (2013).

[7] Sheen, D.M.; McMakin, D.L. and Hall, T.E., "Three-Dimensional Millimeter-Wave Imaging for Concealed Weapon Detection”, IEEE Trans. MTT, vol. 49, no. 9, September, (2001).

[8] Born, M., and Wolf, E.,"Principles of optics”, Cambridge University Press, 7th Edition, (2003).

[9] Goodman, "Introduction to Fourier Optics", 3rd Ed., Roberts \& Company, (2005).

[10] Lipson, A., "Optical Physics", $4^{\text {th }}$ Ed., Cambridge University Press, (2011).

[11] Thomson, A., Moran, M., Swenson, G, "Interferometry and Synthesis in Radio Astronomy”, Wiley, (2004)

[12] Salmon, N. A. "Extended sources near-field processing of experimental aperture synthesis data and application of the Gerchberg method for enhancing radiometric three-dimensional millimetre-wave images in security screening portals", Millimetre Wave and Terahertz Sensors and Technology X, Warsaw, SPIE Proc. vol. 10439, (2017).

[13] Xiangzhou Guo, Muhammad Asif, Anyong Hu, and Jungang Miao, "Design of a low-cost cross-correlator system for aperture synthesis passive millimeter wave imager", Proc. SPIE vol. 10800-3, Europe Security+Defence, Millimetre Wave and Terahertz Sensors and Technology XI, Berlin, September, (2018). 